\title{
COMPETÊNCIA SOCIAL DE PSICÓTICOS: O COMPORTAMENTO DE ALTERNÂNCIA DA FALA EM SITUAÇÕES DE FAZER E RECEBER CRÍTICAS
}

\author{
PSYCHOTICS' SOCIAL SKILLS: INTERACTION \\ BEHAVIOR IN SOCIAL SITUATIONS OF GIVING \\ AND RECEIVING FEEDBACKS
}

\author{
Marina BANDEIRA ${ }^{1}$ \\ Sabrina Martins BARROSO² \\ Fernanda Vale REIS ${ }^{2}$ \\ Tais Ribeiro GASPAR ${ }^{2}$ \\ Magali Milene SILVA²
}

\begin{abstract}
RESUMO
Investigou-se o comportamento de alternância da fala de 35 participantes psicóticos comparativamente a 35 participantes não-clínicos. Dois observadores treinados observaram-nos interagindo com interlocutores treinados em quatro situações sociais de fazer e receber críticas em desempenho de papéis gravados em vídeo. Variaram-se o tipo de situação e o gênero do interlocutor. Os resultados significativos mostraram que o grupo clínico deixou de alternar sua fala com a do interlocutor com mais freqüência do que o grupo não-clínico e teve mais alternâncias curtas, monossilábicas e apenas com sons, assim como menor número de palavras por alternância. O grupo clínico apresentou maior proporção de alternâncias descontextualizadas e de conteúdo verbal insuficiente, assim como maior número de pausas. Ambos os grupos apresentaram mais distúrbios no momento da alternância ao receber do que ao fazer críticas e alternâncias mais longas frente ao interlocutor masculino. Esses resultados replicam dados de outros pesquisadores em outros contextos culturais e indicam quais dimensões específicas da alternância da fala necessitam ser focalizadas no treinamento de habilidades sociais de pacientes psiquiátricos.

Palavras-chave: competência social; psicóticos; inclusão social; desempenho de papéis, pacientes psiquiátricos.
\end{abstract}

\footnotetext{
${ }^{1}$ Departamento de Psicologia, Universidade Federal de São João del Rei. Praça Dom Helvécio, 74, 36300-000, São João del Rei, MG, Brasil. Correspondência para/Correspondence to: M. BANDEIRA. E-mail: <bandeira@ufsj.edu.br>.

${ }^{2}$ Acadêmicas, Curso de Graduação em Psicologia, Universidade Federal de São João Del Rei. São João Del Rei, MG, Brasil. Bolsistas de Iniciação Científica - CNPq. Pesquisa financiada: FAPEMIG E CNPq.
} 


\begin{abstract}
This research has investigated the interaction behavior of 35 psychotics compared to 35 non-clinical participants. Two trained judges have observed them interacting with trained partners in 4 negative assertion role-play situations, regarding giving and receiving criticism, which were videotaped. Types of situations and gender of partners were varied during the procedure. Results indicated the clinical group failed to alternate its speech with its partners more often than the non-clinical group. The clinical group showed greater frequency of very short verbal or vocal responses to their partners interactions. The content of their responses were more totally or partially bizarre. Both groups presented more disruptions regarding the timing of their responses in situations where they were receiving rather then giving criticism. Both groups gave longer verbal responses to male rather than female partners. These results in general replicated data from others researchers and indicated the specific dimensions of interaction behavior need to be addressed in social skills training for psychiatric patients.
\end{abstract}

Key-words: competence (social); psychotics; social inclusion; role play; psychiatric patients.

Em uma interação social, a capacidade de a pessoa alternar adequadamente suas falas com as de seu interlocutor é uma das habilidades sociais importantes para uma comunicação adequada. A alternância tem sido definida como uma troca bem sincronizada de tomadas de palavras e de pausas intercaladas por dois interlocutores em uma interação social (Mueser, Bellack, Douglas \& Morrison, 1991). As pessoas que apresentam déficits em habilidades sociais geralmente demonstram dificuldade na capacidade de alternância, não sabendo julgar por exemplo, o momento apropriado para tomar a palavra e responder verbalmente ao interlocutor (Fischetti, Curran \& Wessberg, 1977).

Comparando pessoas socialmente competentes e incompetentes, Fischetti et al. (1977) constataram que elas diferiam principalmente na habilidade de situar suas falas em momentos adequados da interação social. Mueser et al. (1991) estabeleceram três critérios básicos para definir uma resposta de alternância inadequada: 1) a ocorrência de respostas verbais muito demoradas após a fala do interlocutor, 2) ou, ao contrário, respostas verbais rápidas demais que interrompem a fala do interlocutor, não permitindo que este finalize seu discurso e 3 ) respostas verbais que apresentam conteúdos bizarros que não estão em sintonia com o conteúdo da conversação.

Segundo Mueser et al. (1991), os esquizofrênicos, em particular, possuem dificuldades na habilidade de alternância, o que pode ter um efeito crítico nas interações sociais, uma vez que limita sua capacidade de se comunicar com o outro. A inabilidade dos esquizofrênicos, de alternar adequadamente com o interlocutor e de estabelecer qualquer tipo de sistema de interação ou sincronização foi destacada igualmente por Argyle (1994). Os esquizofrênicos, após a fala do interlocutor, apresentam freqüentemente uma resposta atrasada ou de silêncio, ou seja, uma latência de resposta maior ou até ausência de resposta comparativamente a um grupo não-clínico (Argyle, 1994).

Essa dificuldade de alternância tem sido explicada como resultante de déficits no processo de percepção social e na habilidade cognitiva de processamento de informações dos estímulos que ocorrem em uma interação social (Trower, 1980; Mueser et al.,1991). Estudos experimentais referentes a essa habilidade mostraram que, normalmente, no momento da alternância entre dois interlocutores, ocorrem sinais indicadores 
da alternância (change over signals) - mudanças visuais, de postura e de posição da cabeça da pessoa que fala - que precedem a troca de tomadas de palavras (Kendon, 1967; Argyle, 1984). Esses sinais emitidos pela pessoa que fala seriam, portanto, percebidos pelo interlocutor e teriam a função de the indicar o momento adequado para a tomada de palavras. Considerando a dificuldade de percepção de estímulos sociais apresentada pelos pacientes psicóticos, em particular os esquizofrênicos, esses sinais de alternância poderiam não estar sendo processados por eles, o que dificultaria a emissão do comportamento de alternar no momento adequado.

Mueser et al. (1991) observaram que 14\% dos pacientes psiquiátricos estudados por eles, durante o período de um ano, apresentaram um déficit consistente na habilidade de alternância da fala. Seus escores desse comportamento se situaram consistentemente abaixo da faixa de valores obtidos pelo grupo não-clínico, com o qual foi comparado. Mueser et al. (1991) confirmaram que a alternância foi a única habilidade específica que apresentou um déficit consistente e estável nos mesmos pacientes durante todo o período de um ano de observação.

Esses estudos apontam para déficits específicos dos pacientes psiquiátricos e essa informação tem servido para orientar os programas de treinamento das habilidades sociais dessa população-alvo. Esse tipo de treinamento tem sido freqüentemente utilizado na readaptação psicossocial de pacientes psiquiátricos, visando capacitá-los para interagir com as pessoas de sua comunidade. Diversos programas de readaptação psicossocial e de acompanhamento intensivo dos pacientes na comunidade adotaram esse treinamento como parte integrante de seus procedimentos de intervenção (Stein \& Test, 1980; Liberman, 1984; 1986; Liberman et al., 1993; Bandeira, Gelinas \& Lesage, 1998). Um impacto positivo tem sido observado na reinserção social dos pacientes em função da utilização do treinamento de habilidades sociais em termos de uma redução da freqüência e da duração das reinternações psiquiátricas (Donahoe \& Drisienga,
1988; Hogarty et al., 1991; Wallace, Liberman, Mackain, Blackwell, \& Eckman, 1992).

Pesquisas sobre as habilidades sociais têm sido desenvolvidas com o objetivo de identificar as características principais do comportamento dos pacientes psiquiátricos nas interações sociais, assim como seus principais déficits comportamentais e cognitivos comparativamente a um grupo não-clínico. Kazdin (1977) tem enfatizado a necessidade de se estabelecer parâmetros comparativos, estudando-se grupos não-clínicos que sejam do mesmo nível socioeconômico e da mesma subcultura dos pacientes.

Dentre as estratégias metodológicas mais utilizadas nas pesquisas avaliativas sobre as habilidades sociais de pacientes psiquiátricos, destaca-se como a mais adequada para esse tipo de clientela a observação sistemática do comportamento em situações de desempenho de papéis, gravadas em vídeo. Situações variadas são necessárias para se ter uma avaliação adequada das habilidades dos sujeitos, uma vez que as habilidades sociais não é um traço, mas sim um desempenho que pode variar em função do tipo de situação. Esse tipo de medida apresenta validade preditiva, concomitante e discriminativa (Merluzzi \& Biever, 1987; Bellack, Morrison, Mueser \& Wade, 1989; Bellack, Morrison, Mueser, Wade \& Sayers, 1990; Torgrud e Holborn, 1992), assim como fidedignidade (Mueser et al., 1991).

Utilizando essa metodologia, pesquisas têm indicado a presença de déficits específicos no desempenho dos pacientes psiquiátricos em interações sociais (Trower, 1980; Bellack et al., 1989; Mueser et al., 1991; Tremblay, 1992), em termos dos seus componentes verbais, não-verbais e paralingüísticos e em termos das relações entre o comportamento dos pacientes e de seu interlocutor em uma interação social, tal como o comportamento de alternância. Déficits específicos nos aspectos verbais, não-verbais e paralinguísticos do comportamento de pacientes psiquiátricos foram igualmente observados em nosso meio (Bandeira, Cardoso, Fernandes, Resende \& Santos, 1998; Bandeira \& Tremblay, 
1998; Bandeira \& Ireno, 2002; Bandeira, Machado \& Pereira, 2002).

Não foram encontradas, entretanto, no Brasil, pesquisas focalizando o estudo do comportamento de alternância de pacientes psiquiátricos. Esta pesquisa visa preencher essa lacuna, com o objetivo de descrever as características do comportamento de alternância da fala dos pacientes, comparativamente a um grupo não-clínico de sua comunidade, através da observação sistemática do comportamento.

\section{MÉTODO}

Participaram desta pesquisa 70 pessoas do sexo masculino, de baixo nível socioeconômico, residentes em São João del Rei e região, que formavam dois grupos: clínico e não-clínico. O grupo clínico compunha-se de 35 pacientes psiquiátricos desinstitucionalizados, com idade média de 39,4 anos, selecionados a partir dos arquivos do Hospital Psiquiátrico da FHEMIG, em Barbacena, e do Hospital Paulo Menicucci, em Lavras, e diagnosticados pelos psiquiatras dos hospitais como CID-295 e CID-298. Todos os participantes do grupo clínico estavam sendo medicados com drogas antipsicóticas no período da pesquisa. O grupo não-clínico era composto de 35 participantes, moradores da mesma região da cidade que os pacientes, com idade média de 37,4 anos, sem história psiquiátrica.

O estudo da alternância foi realizado através da observação sistemática do comportamento dos participantes em situações de desempenho de papéis que representavam interações sociais cotidianas, gravadas em vídeo.

Os desempenhos de papéis foram feitos com base na Escala de Avaliação de Competência Social (EACS) (Bandeira, 2002), adaptada e validada para o Brasil a partir de uma escala canadense de competência social (Tremblay \& Bandeira, 1989). A EACS consiste em um sistema de avaliação do comportamento através da observação sistemática em quatro situações sociais cotidianas nas quais são variados o gênero do interlocutor e as demandas das situações. A EACS inclui ainda a descrição dos cenários a serem utilizados em cada situação, os tipos de réplicas a serem usadas pelos interlocutores e as instruções a serem dadas aos participantes antes de iniciar os desempenhos de papéis, assim como as descrições dos critérios de avaliação da competência social em uma escala de medidas seis pontos crescentes quanto ao grau de competência social (Bandeira, 2002).

Para o estudo das interações sociais, foi utilizada uma filmadora para registrar o comportamento dos participantes e de seus interlocutores nos desempenhos de papéis, um aparelho de videocassete e uma televisão para observar os comportamentos dos participantes, assim como um cronômetro e folhas de papel para registrar as categorias de alternância estudadas. As folhas de registro incluíam as categorias estudadas, descritas abaixo, nas quais os observadores anotavam suas observações.

\section{Situações sociais}

As quatro situações de desempenho de papéis utilizadas incluíam duas situações em que o participante iniciava uma interação e outras duas nas quais ele reagia ao comportamento do interlocutor. Nas situações de iniciar a interação, o participante devia expressar a sua insatisfação diante do comportamento do interlocutor e solicitar uma mudança do seu comportamento. Nas outras duas situações, ele devia receber uma crítica justificada do interlocutor a respeito de seu próprio comportamento, aceitando sua responsabilidade, mas também expressando sua necessidade naquela situação e fornecendo uma solução que atendesse suas necessidades sem desrespeitar a do interlocutor. As situações sociais eram conduzidas por interlocutores previamente treinados para esse fim, os quais utilizavam réplicas múltiplas padronizadas, porém flexíveis, que favoreciam uma maior naturalidade nas interações sociais (Bandeira, 2002). 


\section{Categorias de comportamento observadas}

As medidas utilizadas para avaliar as categorias do comportamento de alternância da fala dos participantes, nas interações sociais estudadas, estão descritas abaixo:

- Réplicas respondidas e não-respondidas: 1) número de réplicas respondidas - número de vezes em que o participante respondia às falas do interlocutor, portanto, sem que ocorressem silêncios do participante como resposta; 2) número de réplicas não respondidas - número de vezes em que o participante silenciava após a fala do interlocutor, não apresentando nenhuma resposta;

- Duração das alternâncias: 1) proporção de alternâncias monossilábicas - número de vezes em que o participante respondia ao interlocutor com um monossílabo, dividido pelo número total de réplicas respondidas; 2) proporção de alternâncias curtas - número de vezes em que o participante respondia ao interlocutor usando até cinco palavras, dividido pelo número total de réplicas respondidas; 3 ) proporção de alternâncias apenas com sons - número de vezes em que o participante respondia ao interlocutor emitindo apenas sons, tais como ham-ham, hum-hum, dividido pelo número total de réplicas respondidas; 4) número de palavras - soma do número de palavras emitidas pelo participante em cada uma de suas alternâncias; 5) número médio de sons - soma do número de sons emitidos pelo participante em resposta à fala do interlocutor, dividido pelo número de réplicas respondidas; 6 ) tempo médio das alternâncias - soma dos intervalos de tempo, em segundos e centésimos de segundo, de cada alternância do sujeito em resposta a cada fala do interlocutor, dividido pelo número de réplicas respondidas;

- Ritmo das alternâncias: 1) proporção de alternâncias com pausas - número de vezes em que o participante apresentava alternâncias nas quais ele fazia silêncios de mais de três segundos no decorrer de sua fala, dividido pelo número total de réplicas respondidas; 2 ) número médio de pausas - número de vezes em que o participante fazia silêncios superiores a três segundos durante sua fala, dividido pelo número de alternâncias; 3 ) proporção da duração das pausas - intervalo de tempo, medido em segundos, das pausas feitas pelos participantes ao falar, dividido pela duração das alternâncias; 4) proporção de alternâncias com hesitações - número de alternâncias em que o participante demonstrava incerteza, insegurança ou falta de fluência, tais como murmúrios, dúvidas, repetições, dividido pelo número total de alternâncias;

- Momento da alternância: 1) proporção de alternâncias interrompendo o interlocutor - número de vezes em que o participante tomava a palavra sem esperar que o interlocutor terminasse a sua fala, dividido pelo número total de réplicas respondidas; 2) proporção de alternâncias atrasadas - número de vezes em que o participante iniciava sua resposta ao interlocutor após uma latência igual ou superior a três segundos, dividido pelo número total de réplicas respondidas; 3 ) proporção de alternâncias com latência adequada - número de vezes em que o participante iniciava sua resposta ao interlocutor com uma latência inferior a três segundos, sem interromper a fala do interlocutor, dividido pelo número total de réplicas respondidas; 4) número médio de interrupções - número de vezes em que o participante respondia ou tentava responder ao interlocutor, sem que ele tivesse terminado a sua fala, dividido pelo número de alternâncias; 5) duração média da latência - soma dos intervalos de tempo, em segundos e centésimos de segundo, de cada latência do sujeito ao responder a cada fala do interlocutor, dividido pelo número de réplicas respondidas;

- Contextualização verbal das alternâncias: 1) proporção de alternâncias totalmente descontextualizadas - número de alternâncias que diferiam do conteúdo previamente mencionado pelo interlocutor ou do assunto tratado, de modo a perturbar o sentido da conversação, dividido pelo número total de réplicas respondidas; 2 ) proporção de alternâncias parcialmente descontextualizadas - número de alternâncias em que o participante evitava o conteúdo do assunto tratado, evitava responder, mas ainda se mantinha 
dentro do tema tratado, dividido pelo número total de réplicas respondidas; 3 ) proporção de alternâncias contextualizadas - número de alternâncias em que o participante apresentava conteúdo verbal coerente com a fala anterior do interlocutor e com o tema tratado, dividido pelo número total de réplicas respondidas;

- Contextualização não-verbal das alternâncias: 1) proporção de alternâncias descontextualizadas - número de alternâncias em que o participante apresentava comportamento não-verbal incoerente com a situação, dividido pelo número total de réplicas respondidas; 2) proporção de alternâncias contextualizadas - número de alternâncias em que o participante apresentava comportamento não-verbal coerente com a situação, dividido pelo número total de réplicas respondidas;

- Suficiência do conteúdo: 1) proporção de alternâncias insuficientes - número de alternâncias em que o participante apresentava um conteúdo da fala incompleto, dividido pelo número total de réplicas respondidas; 2) proporção de alternâncias suficientes - número de alternâncias em que o participante apresentava um conteúdo da fala completo, dividido pelo número total de réplicas respondidas; 3) proporção de alternâncias prolixas - número de vezes em que a resposta dada pelo participante ao interlocutor era demasiadamente extensa ou repetitiva, prejudicando o curso da interação, dividido pelo número total de réplicas respondidas;

- Cotas de adequação das alternâncias: avaliação do grau de adequação do comportamento dos participantes de tomar a palavra e alternar sua fala com a do interlocutor, em termos do momento, do ritmo e do conteúdo da alternância, medidos através de uma escala tipo Likert de quatro pontos ou cotas, sendo 10 menor valor de adequação e 4 o maior valor de adequação;

- Competência social: avaliação do grau de competência social dos participantes em cada situação estudada, em termos da impressão global do comportamento apresentado, através de uma escala tipo Likert de seis pontos, sendo 1 muito incompetente e 6 muito competente.
Essa avaliação já havia sido feita em um trabalho anterior por dois observadores independentes que desconheciam, portanto, os dados referentes ao comportamento de alternância dos sujeitos.

\section{Procedimento}

Os participantes eram convocados pessoalmente a participar da pesquisa, recebiam informações sobre os objetivos do trabalho, assinavam um termo de consentimento esclarecido, eram acompanhados até o local da pesquisa e recebiam instruções para sua participação em cada situação de interação social. As instruções incluíam a descrição da situação e a solicitação para se comportarem naturalmente, como fariam na vida cotidiana. As sessões ocorriam em uma sala especialmente equipada com os cenários necessários aos desempenhos de papéis. Eram eliminados os participantes que não compreendiam as instruções recebidas.

O comportamento dos participantes foi observado por dois pares de juízes previamente treinados. O primeiro par fazia a observação de algumas categorias de comportamento no período da manhã e o segundo par observava outras categorias diferentes no período da tarde.

Os juízes foram treinados pelo pesquisador para observar, de forma precisa e fidedigna, o comportamento dos participantes interagindo com um interlocutor nas situações sociais estudadas. Para o treinamento, foram utilizadas as gravações de pessoas que não estavam incluídas na pesquisa.

Os juízes observavam diariamente o comportamento dos participantes, gravado em vídeo, utilizando uma folha de registro dos componentes observados. Os juízes faziam suas observações independentemente um do outro. Após a observação feita pelo primeiro juiz de um sujeito, nas quatro situações, o segundo juiz observava o mesmo sujeito. Após a sessão de observação, os dois juízes se reuniam com o pesquisador para discutir as possíveis causas dos desacordos obtidos entre eles, buscando estabelecer definições mais objetivas sobre os 
comportamentos observados e definir critérios comuns para a observação que minimizassem a ocorrência de desacordos. Ambos passavam então a adotar os mesmos critérios para a observação daqueles itens sobre os quais haviam discordado. O treinamento foi feito até que os juízes atingiram uma porcentagem mínima de $85 \%$ de acordo entre eles, calculada a partir do número de categorias em que houve acordo entre eles em relação ao total de categorias observadas.

Após o treinamento dos juízes, procedeu-se à coleta dos dados. Os juízes observavam, em vídeo, apenas um participante por dia, a fim de evitar que o cansaço provocasse um aumento de erros de observação. Os juízes continuaram a fazer as suas observações independentemente um do outro, tal como descrito acima.

Os juízes observavam, em vídeo, cada componente do comportamento do participante separadamente, seguindo-se a ordem da folha de registro de comportamentos. Observava-se 0 primeiro componente e, em seguida, rebobinava-se a fita para observar e registrar o segundo componente na mesma situação e assim por diante. Esse procedimento visava facilitar a concentração dos observadores sobre um único aspecto do comportamento do sujeito, de forma a diminuir o risco de erros de observação devidos à distração.

Após a observação do comportamento de um participante, os dois juízes iniciavam uma reunião de consenso, na qual calculavam a porcentagem de acordos e desacordos obtidos. Quando havia desacordo, discutia-se e chegava-se a um consenso que resultava na medida final do comportamento. No caso das medidas de duração de um comportamento (ex.: duração de cada alternância do sujeito), considerava-se como acordo, quando a diferença entre as medidas dos dois juízes, expressas em segundos e centésimos de segundo, se situava dentro da faixa de apenas $15 \%$ de diferença entre eles. Caso a diferença fosse superior a $15 \%$, considerava-se como desacordo e o consenso era obtido calculando-se a média das medidas dos dois juízes. Para as medidas de freqüência, os juízes refaziam juntos a observação do comportamento em questão, discutindo sobre as causas dos desacordos até que chegassem a um consenso final, levando em consideração as definições dos comportamentos. Em seguida, os dados do consenso eram anotados em uma folha específica para esse fim.

Para se obter uma maior fidedignidade das medidas, foram adotados os seguintes procedimentos de coleta de dados. No caso das medidas de tempo, cada juiz cronometrava sempre três vezes o comportamento observado e, em seguida, adotava a mediana dessas três medidas. Esse procedimento visava diminuir o número de desacordos entre os juízes, uma vez que uma pequena diferença de centésimos de segundo nas medidas de duração era suficiente para ultrapassar a margem dos $15 \%$ exigidos para se considerar como acordo. No caso de algumas medidas de freqüência de comportamento que implicavam em maior dificuldade para os observadores (ex. número de alternâncias com interrupções), adotou-se esse mesmo procedimento.

\section{RESULTADOS}

\section{Comparação entre os grupos}

Para os resultados das comparações entre os grupos clínico e não-clínico, através da Análise de Variância, com relação às categorias da alternância estudadas (Tabela 1), pode-se observar que o grupo clínico apresentou uma média significativamente maior de réplicas não respondidas do que o grupo não-clínico ( $\mathrm{F}=4,03$; $p<0,05)$, ou seja, os participantes pacientes deixaram de alternar suas falas com as do interlocutor mais freqüentemente.

Nas ocasiões em que alternaram sua fala com a do interlocutor, os participantes pacientes apresentaram uma proporção significativamente maior de alternâncias curtas de até cinco palavras $(\mathrm{F}=11,77 ; p<0,001)$, de alternâncias monossilábicas $(F=5,85 ; p<0,05)$ e de alternâncias apenas com sons $(F=5,74 ; p<0,05)$ do que o grupo não-clínico. Essa predominância de respostas 
reduzidas se refletiu também na medida de número de palavras usadas por alternância, que foi significativamente menor para o grupo clínico $(\mathrm{F}=6,05 ; p<0,05)$, assim como na duração das alternâncias, medidas em segundos, que foi significativamente menor para o grupo clínico $(\mathrm{F}=4,23 ; p<0,05)$. As respostas verbais prolixas foram igualmente infreqüentes nos dois grupos. Os aspectos relativos ao momento de alternar também não apresentaram diferenças significativas entre os grupos (Tabela 1).

No que se refere ao conteúdo da fala, pode-se observar (Tabela 1) que os participantes pacientes apresentaram uma proporção significativamente menor de alternâncias completamente contextualizadas $(F=13,62$; $p<0,001)$. Ao contrário, eles apresentaram uma proporção significativamente maior de alternâncias parcialmente descontextualizadas $(F=8,23$; $p<0,05)$ e totalmente descontextualizadas $(\mathrm{F}=8,50 ; p<0,05)$. Quanto à contextualização do aspecto não-verbal das alternâncias, não foi observada diferença significativa entre os grupos.

Pode-se observar, ainda (Tabela 1 ), que os participantes pacientes alternaram com 0 interlocutor mais freqüentemente usando conteúdos insuficientes ( $F=18,05 ; p<0,001)$ e menos freqüentemente usando conteúdos suficientes ( $F=16,52 ; p<0,001)$, mostrando assim um padrão de resposta verbal que apresentava de forma incompleta seu argumento ou opinião ao interlocutor.

No que se refere ao ritmo da alternância, pode-se observar também que os participantes pacientes apresentaram um número significativamente maior de pausas por alternância $(F=4,10$; $p<0,05)$. Não houve diferença significativa entre os grupos com relação às medidas globais de grau de adequação da alternância.

A importância relativa das variáveis descritas acima foi analisada através da Análise Discriminante, a fim de identificar quais eram os aspectos da alternância que mais diferenciavam os grupos clínico e não-clínico. Os resultados mostraram uma função discriminante estatisticamente significativa $\left(\chi^{2}=19,53 ; p=0,007\right.$; Wilks' Lambda $=0,72)$. Os coeficientes de correlação canônicos (c.c.) obtidos entre as variáveis da alternância e a função discriminante mostraram que os aspectos que mais diferenciavam os pacientes do seu grupo de referência, em ordem decrescente de importância, foram: a insuficiência do conteúdo da sua fala (c.c. $=0,86$ ), a baixa proporção de alternâncias com conteúdos contextualizados (c.c. $=-0,74$ ), a curta duração das suas alternâncias (c.c. $=0,59$ ), o baixo número de palavras durante suas alternâncias (c.c.=-0,44), a freqüência maior de pausas por minuto (c.c. $=0,39)$ e a freqüência maior do comportamento de não alternar sua fala com a do interlocutor (c.c. $=0,36)$.

Considerando que o comportamento de alternância constitui um dos componentes da competência social, foi feita uma análise correlacional de Pearson entre as medidas dessas duas variáveis.

Os resultados mostraram que todas as categorias da alternância estudadas na presente pesquisa estavam correlacionadas significativamente com o grau de competência social. Observou-se que quanto maior era o grau de competência social dos participantes menor era a freqüência de conteúdos insuficientes na sua fala $(r=-0,61 ; p<0,001)$, ou seja, mais completa era a expressão verbal da sua opinião ao falar com o interlocutor. Além disso, quanto maior a competência social dos participantes, mais contextualizado era o conteúdo de sua fala $(r=0,58 ; p<0,001)$, menos frases curtas eles utilizavam $(r=-0,46 ; p<0,001)$, menos pausas por minuto eles faziam $(r=-0,49 ; p<0,001)$, maior número de palavras eles utilizavam, maior era a duração em segundos de sua fala $(r=0,42$; $p<0,001)$ e maior era o número de réplicas feitas por eles $(r=0,39 ; p<0,001)$. Observaram-se, portanto, relações significativas entre as categorias estudadas da alternância e a impressão global de competência social veiculada pelos participantes nas interações sociais. 
Tabela 1. Médias, proporções, cotas de adequação e desvios-padrão das categorias de alternância, dos grupos clínico e não-clínico.

\begin{tabular}{|c|c|c|c|c|c|c|}
\hline \multirow{2}{*}{ Categorias de alternância } & \multicolumn{3}{|c|}{ Grupo Clínico } & \multicolumn{3}{|c|}{ Grupo Não-clínico } \\
\hline & Média & \pm & $\mathrm{DP}$ & Média & \pm & DP \\
\hline \multicolumn{7}{|l|}{ Réplicas respondidas e não respondidas } \\
\hline Réplicas respondidas & 17,70 & \pm & 3,89 & 17,91 & \pm & 2,99 \\
\hline Réplicas não respondidas & 1,59 & \pm & 2,66 & 0,56 & \pm & $1,35^{\star}$ \\
\hline \multicolumn{7}{|l|}{ Momento das alternâncias } \\
\hline Proporção de alternâncias interrompendo (\%) & 9,70 & \pm & 0,11 & 14,00 & \pm & 0,09 \\
\hline Proporção de alternâncias atrasadas (\%) & 8,10 & \pm & 0,11 & 6,40 & \pm & 0,08 \\
\hline Proporção de alternâncias com latência adequada (\%) & 81,00 & \pm & 0,16 & 81,00 & \pm & 0,10 \\
\hline Número médio de interrupções & 2,00 & \pm & 2,49 & 2,94 & \pm & 2,39 \\
\hline Duração média da latência (segundos) & 0,90 & \pm & 0,52 & 0,93 & \pm & 0,47 \\
\hline \multicolumn{7}{|l|}{ Duração das alternâncias } \\
\hline Proporção de alternâncias monossilábicas (\%) & 8,20 & \pm & 0,07 & 4,20 & \pm & $0,06^{*}$ \\
\hline Proporção de alternâncias curtas (\%) & 42,00 & \pm & 0,18 & 28,00 & \pm & $0,15^{\star *}$ \\
\hline Proporção de alternâncias apenas com sons (\%) & 3,00 & \pm & 0,04 & 1,00 & \pm & $0,03^{*}$ \\
\hline Número de palavras & 219,35 & \pm & 73,24 & 336,44 & \pm & $16,74^{*}$ \\
\hline Número médio de sons & 4,82 & \pm & 3,98 & 3,53 & \pm & 2,98 \\
\hline Tempo médio (segundos) & 4,27 & \pm & 3,47 & 6,02 & \pm & $3,55^{\star}$ \\
\hline \multicolumn{7}{|l|}{ Contextualização verbal } \\
\hline Contextualizadas (\%) & 81,00 & \pm & $0,19 * *$ & 93,00 & \pm & 0,082 \\
\hline Parcialmente descontextualizadas (\%) & 13,00 & \pm & 0,13 * & 5,00 & \pm & 0,07 \\
\hline Totalmente descontextualizadas (\%) & 5,00 & \pm & $0,09 *$ & 0,30 & \pm & 0,01 \\
\hline \multicolumn{7}{|l|}{ Contextualização não-verbal } \\
\hline Proporção de alternância contextualizadas (\%) & 94,70 & \pm & 0,12 & 96,00 & \pm & 0,12 \\
\hline Proporção de alternância descontextualizadas (\%) & 5,00 & \pm & 0,11 & 4,00 & \pm & 0,12 \\
\hline \multicolumn{7}{|l|}{ Suficiência do conteúdo } \\
\hline Proporção de alternância suficientes (\%) & 67,00 & \pm & 0,20 ** & 82,00 & \pm & 0,12 \\
\hline Proporção de alternância prolixas (\%) & 7,00 & \pm & 0,11 & 7,00 & \pm & 0,10 \\
\hline Proporção de alternância insuficientes (\%) & 23,00 & \pm & $0,18 * *$ & 8,00 & \pm & 0,10 \\
\hline \multicolumn{7}{|l|}{ Rítmo das alternâncias } \\
\hline Proporção de alternância com pausas (\%) & 2,30 & \pm & 0,04 & 1,50 & \pm & 0,03 \\
\hline Proporção de alternância com hesitações (\%) & 24,00 & \pm & 0,14 & 23,00 & \pm & 0,14 \\
\hline Número médio de pausas & 0,66 & \pm & $1,17^{*}$ & 0,22 & \pm & 0,43 \\
\hline Proporção de duração das pausas (\%) & 4,25 & \pm & 0,07 & 1,64 & \pm & 0,03 \\
\hline \multicolumn{7}{|l|}{ Cota de adequação (1 a 4) } \\
\hline Adequação global & 2,40 & \pm & 0,80 & 2,62 & \pm & 0,63 \\
\hline Adequação do momento & 2,64 & \pm & 0,86 & 2,81 & \pm & 0,63 \\
\hline Adequação do ritmo & 2,92 & \pm & 0,71 & 3,05 & \pm & 0,56 \\
\hline
\end{tabular}

${ }^{*} p<0,05 ;{ }^{* *} p<0,001$. 
Tabela 2. Médias, proporções, cotas de adequação e desvios-padrão das categorias de alternância dos grupos clínico e não-clínico por tipo de situação.

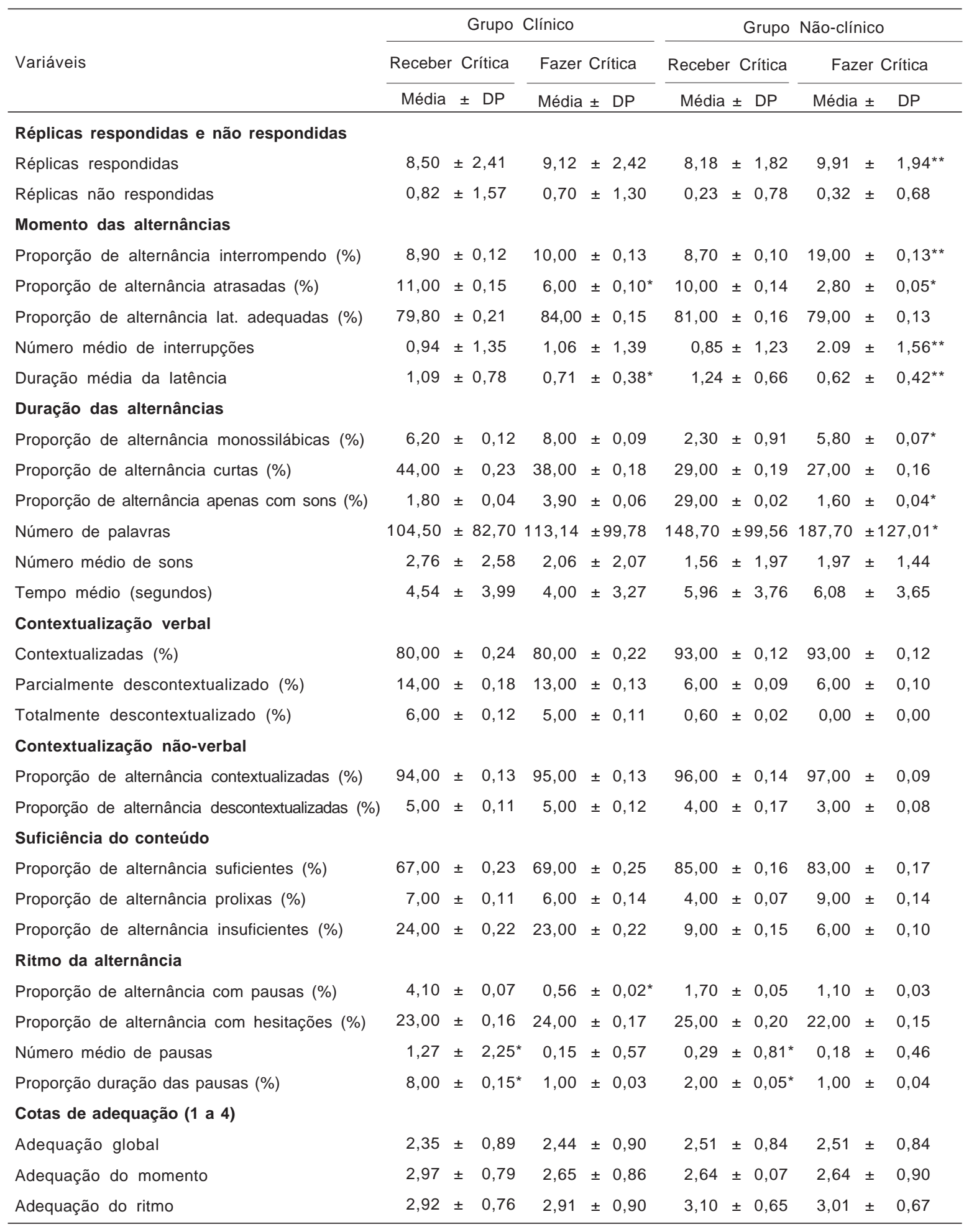

${ }^{*} p<0,05 ;{ }^{* *} p<0,001$ 
No que se refere aos tipos de demandas das situações, as reações dos participantes frente a situações de fazer e de receber críticas foram analisadas separadamente para cada grupo (Tabela 2). Pode-se observar que ambos os grupos apresentaram principalmente distúrbios referentes ao momento e ao ritmo das alternâncias nas situações de receber críticas. Os participantes pacientes apresentaram maior proporção de alternâncias com pausas $(\mathrm{t}=3,22 ; p<0,05)$ ao responderem ao interlocutor, maior número de pausas por minuto $(\mathrm{t}=3,24 ; p<0,05)$, e maior duração das pausas ao falar $(\mathrm{t}=3,04 ; p<0,05)$ nas situações de receber crítica do que nas de fazer crítica. Apresentaram também uma proporção significativamente maior de alternâncias atrasadas ( $\mathrm{t}=2,26 ; p<0,05)$ e uma duração maior das latências $(\mathrm{t}=3,23 ; p<0.05)$ ao alternar com o interlocutor nas situações de receber crítica do que na de fazer crítica.

Os participantes do grupo não-clínico apresentaram uma variabilidade maior do comportamento entre os dois tipos de situação, pois houve um maior número de diferenças significativas em diversas dimensões do comportamento de alternância. Os participantes desse grupo apresentaram uma maior proporção de alternâncias atrasadas $(t=3,05 ; p<0,05)$ e maior duração das latências de resposta ( $\mathrm{t}=5,86$; $p<0,001)$ nas situações de receber crítica. Apresentaram também maior duração das pausas ao falar $(\mathrm{t}=3,04 ; p<0,05)$ nas situações de receber do que nas de fazer crítica. O grupo não-clínico apresentou ainda uma proporção muito maior de alternâncias apenas com vocalizações ( $\mathrm{t}=-2,21 ; p<0,05)$ e uma proporção menor de alternâncias monossilábicas ( $\mathrm{t}=-2,44$; $p<0,05)$ nas situações de receber crítica. Além disso, foi nas situações de receber crítica que esse grupo apresentou uma proporção menor de alternâncias respondidas ( $\mathrm{t}=-5,01 ; p<0,001)$, de alternâncias com interrupções da fala do interlocutor ( $\mathrm{t}=-4,07 ; p<0,001)$ e um número menor de interrupções por fala $(t=-4,80 ; p<0,05)$. Foi igualmente ao receber crítica que os participantes desse grupo apresentaram menor número de palavras em suas alternâncias ( $\mathrm{t}=$ $3,18 ; p<0,001)$. Ou seja, os participantes desse grupo também foram mais parcimoniosos nas situações de receber crítica do que nas de fazer crítica.

\section{Comparação entre os tipos de interlocutores masculino e feminino}

Pode-se observar (Tabela 3) que, para ambos os grupos, houve uma menor variabilidade frente a mudanças no gênero do interlocutor, já que se obteve um menor número de diferenças significativas entre essas situações do que se observou para as mudanças nas demandas das situações.

No que se refere ao grupo clínico (Tabela 3), foi observado que os participantes apresentaram uma menor proporção de alternâncias curtas $(t=2,09 ; p<0,05)$ e maior proporção de alternâncias no momento adequado $(t=-2,25$; $p<0,05)$ frente ao interlocutor masculino do que frente ao feminino. Nenhuma outra dimensão estudada apresentou diferença significativa.

Quanto ao grupo não-clínico, os participantes apresentaram maior variabilidade comportamental frente ao gênero do interlocutor. Os participantes desse grupo apresentaram uma menor proporção de alternâncias curtas ( $\mathrm{t}=3,18$; $p<0,05)$ e monossilábicas $(t=2,44 ; p<0,05)$ frente ao interlocutor masculino. O grupo não-clínico apresentou ainda maior proporção de alternâncias no momento adequado $(\mathrm{t}=-3,96 ; p<0,001) \mathrm{e}$ menor proporção de alternâncias com interrupções $(\mathrm{t}=4,56 ; p<0,001)$, assim como um menor número de interrupções por fala $(t=2,71 ; p<0,05)$ frente ao interlocutor masculino. Além disso, o grupo não-clínico apresentou uma duração maior da fala $(\mathrm{t}=-2,45 ; p<0,05)$ ao tomar a palavra junto ao interlocutor masculino.

\section{DISCUSSÃO E CONCLUSÃO}

Os resultados obtidos no presente estudo mostraram, em primeiro lugar, que os participantes 
Tabela 3. Médias, proporções, cotas de adequação e desvios-padrão, das categorias de alternância dos grupos clínico e não-clínico, em função do gênero do interlocutor.

\begin{tabular}{|c|c|c|c|c|c|c|c|c|c|c|}
\hline & \multicolumn{4}{|c|}{ Grupo Clínico } & \multicolumn{6}{|c|}{ Grupo Não-clínico } \\
\hline & \multicolumn{2}{|c|}{ Feminino } & \multicolumn{2}{|c|}{ Masculino } & \multicolumn{3}{|c|}{ Feminino } & \multicolumn{3}{|c|}{ Masculino } \\
\hline & Média \pm & $\mathrm{DP}$ & Média \pm & DP & Média & \pm & DP & Média & \pm & DP \\
\hline \multicolumn{11}{|l|}{ Réplicas respondidas e não respondidas } \\
\hline Réplicas respondidas & $8,70 \pm$ & 2,22 & $8,91 \pm$ & 2,50 & 9,09 & \pm & 1,97 & 9,00 & \pm & 2,09 \\
\hline Réplicas não respondidas & $0,88 \pm$ & 1,68 & $0,70 \pm$ & 1,06 & 0,29 & \pm & 0,94 & 0,26 & \pm & 0,66 \\
\hline \multicolumn{11}{|l|}{ Momento das alternâncias } \\
\hline Proporção de alternância interrompendo (\%) & $12,00 \pm$ & 0,14 & $8,30 \pm$ & 0,15 & 20,00 & \pm & 0,13 & 8,80 & \pm & $0,11^{* *}$ \\
\hline Proporção de alternância atrasadas (\%) & $10,00 \pm$ & 0,15 & $6,30 \pm$ & 0,11 & 6,90 & \pm & 0,10 & 5,70 & \pm & 0,09 \\
\hline Proporção de alternância lat. adequada (\%) & $78,00 \pm$ & 0,18 & $85,00 \pm$ & $0,20 *$ & 73,00 & \pm & 0,16 & 85,00 & \pm & $0,13^{* *}$ \\
\hline Número médio interrupções & $1,06 \pm$ & 1,47 & $0,91 \pm$ & 1,58 & 1,79 & \pm & 1,36 & 1,06 & \pm & $1,41^{*}$ \\
\hline Duração média da latência & $0,95 \pm$ & 0,67 & $0,85 \pm$ & 0,47 & 0,95 & \pm & 0,60 & 0,92 & \pm & 0,51 \\
\hline \multicolumn{11}{|l|}{ Duração das alternâncias } \\
\hline Proporção de alternância monossilábicas (\%) & $9,30 \pm$ & 0,11 & $5,90 \pm$ & 0,08 & 6,70 & \pm & 0,12 & 1,90 & \pm & $0,04^{*}$ \\
\hline Proporção de alternância curtas (\%) & $45,00 \pm$ & 0,23 & $37,00 \pm$ & 0,20 * & 32,00 & \pm & 0,19 & 22,00 & \pm & $0,16^{*}$ \\
\hline Proporção de alternância apenas com sons (\%) & $2,90 \pm$ & 0,06 & $2,80 \pm$ & 0,06 & 1,20 & \pm & 0,03 & 0,59 & \pm & 0,02 \\
\hline Número de palavras & $101,35 \pm 8$ & 86,39 & $116,44 \pm$ & 95,01 & 158,47 & \pm & 120,40 & 179,29 & & 06,73 \\
\hline Número médio de sons & $2,32 \pm$ & 2,62 & $2,50 \pm$ & 2,50 & 2,09 & \pm & 1,86 & 1,68 & \pm & 1,61 \\
\hline Tempo médio (segundos) & $4,08 \pm$ & 3,72 & $4,46 \pm$ & 3,64 & 5,49 & \pm & 3,80 & 6,55 & \pm & $3,73^{\star}$ \\
\hline \multicolumn{11}{|l|}{ Contextualização verbal } \\
\hline Contextualizadas (\%) & $79,00 \pm$ & 0,22 & $80,00 \pm$ & 0,23 & 94,00 & \pm & 0,09 & 92,00 & \pm & 0,13 \\
\hline Parcialmente descontextualizadas (\%) & $14,00 \pm$ & 0,17 & $12,00 \pm$ & 0,18 & 5,00 & \pm & 0,09 & 7,00 & \pm & 0,12 \\
\hline Totalmente descontextualizadas (\%) & $6,00 \pm$ & 0,11 & $4,00 \pm$ & 0,11 & 0,60 & \pm & 0,02 & 0,00 & \pm & 0,00 \\
\hline \multicolumn{11}{|l|}{ Contextualização não-verbal } \\
\hline Proporção de alternância contextualizadas (\%) & $94,00 \pm$ & 0,15 & $94,00 \pm$ & 0,12 & 98,00 & \pm & 0,10 & 95,00 & \pm & 0,13 \\
\hline Proporção de alternância descontextualizadas (\%) & $5,00 \pm$ & 0,13 & $5,00 \pm$ & 0,11 & 2,00 & \pm & 0,12 & 5,00 & \pm & 0,14 \\
\hline \multicolumn{11}{|l|}{ Suficiência do conteúdo } \\
\hline Proporção de alternância suficientes (\%) & $67,00 \pm$ & 0,24 & $67,00 \pm$ & 0,21 & 85,00 & \pm & 0,15 & 84,00 & \pm & 0,16 \\
\hline Proporção de alternância prolixas (\%) & $6,00 \pm$ & 0,11 & $7,00 \pm$ & 0,14 & 6,00 & \pm & 0,10 & 7,00 & \pm & 0,14 \\
\hline Proporção de alternância insuficientes (\%) & $26,00 \pm$ & 0,22 & $21,00 \pm$ & 0,20 & 9,00 & \pm & 0,16 & 7,00 & \pm & 0,09 \\
\hline \multicolumn{11}{|l|}{ Rítmo das alternâncias } \\
\hline Proporção de alternância com pausas (\%) & $2,40 \pm$ & 0,05 & $2,20 \pm$ & 0,05 & 1,10 & \pm & 0,03 & 1,80 & \pm & 0,05 \\
\hline Proporção de alternância com hesitações (\%) & $24,00 \pm$ & 0,17 & $24,00 \pm$ & 0,17 & 23,00 & \pm & 0,14 & 24,00 & \pm & 0,20 \\
\hline Número médio de pausas & $0,73 \pm$ & 1,59 & $0,64 \pm$ & 1,51 & 0,27 & \pm & 0,65 & 0,16 & \pm & 0,57 \\
\hline Proporção duração das pausas (\%) & $4,00 \pm$ & 0,10 & $4,00 \pm$ & 0,10 & 2,00 & \pm & 0,06 & 1,00 & \pm & 0,03 \\
\hline \multicolumn{11}{|l|}{ Cotas de adequação (1 a 4) } \\
\hline Adequação global & $2,38 \pm$ & 0,88 & $2,41 \pm$ & 0,89 & 2,58 & \pm & 0,77 & 2,65 & \pm & 0,71 \\
\hline Adequação do momento & $2,55 \pm$ & 0,97 & $2,72 \pm$ & 0,93 & 2,71 & \pm & 0,82 & 2,91 & \pm & 0,71 \\
\hline Adequação do ritmo & $3,00 \pm$ & 0,73 & $2,84 \pm$ & 0,88 & 3,08 & \pm & 0,61 & 3,02 & \pm & 0,72 \\
\hline
\end{tabular}

${ }^{*} p<0,05 ;{ }^{* *} p<0,001$ 
do grupo clínico apresentaram um déficit significativo no comportamento de alternância da fala comparativamente a um grupo não-clínico de mesmo nível socioeconômico e escolaridade e habitando o mesmo meio geográfico dos pacientes. Avaliações comparativas efetuadas desse modo, com grupos de mesmo nível e da mesma subcultura dos pacientes, possibilitam resultados de maior validade social, segundo Kazdin (1977) e Torgrud \& Holborn (1992).

O déficit observado no presente estudo se referiu, primeiramente, a uma ausência de alternâncias, que ocorreu com uma freqüência significativamente maior por parte do grupo clínico. Ou seja, os participantes do grupo clínico deixaram de responder ao interlocutor mais freqüentemente do que os participantes do grupo não-clínico, afetando assim diretamente a alternância da fala, uma vez que a alternância foi definida por Mueser et al. (1991) como uma troca harmoniosa de tomada de palavras entre os dois pólos de uma interação social. O resultado obtido está em acordo com dados de outros pesquisadores que relataram igualmente a ausência de resposta após a fala do interlocutor em pacientes psiquiátricos, em particular os esquizofrênicos (Argyle, 1994).

Além disso, foi observado, no presente trabalho, um déficit em diversas dimensões da alternância, envolvendo a suficiência e a contextualização do conteúdo da fala, assim como a duração e o ritmo das alternâncias. Ou seja, os participantes pacientes apresentaram mais alternâncias com conteúdo descontextualizado em relação ao problema discutido com o interlocutor. Apresentaram também insuficiência do conteúdo de sua fala, fornecendo explicações incompletas ao interlocutor. Observaram-se ainda diferenças no ritmo da alternância da fala; o grupo clínico apresentou mais pausas ao falar do que o grupo não-clínico. Quanto à duração das alternâncias, os participantes pacientes apresentaram mais alternâncias curtas, monossilábicas ou simplesmente envolvendo vocalizações sem palavras, assim como um número muito menor de palavras ao falar. A análise discriminante mostrou que os três principais aspectos que mais diferenciaram o grupo clínico do grupo nãoclínico foi a insuficiência, a descontextualização e a curta duração de suas alternâncias. Esses resultados mostram, portanto, um déficit referente a diversas dimensões da alternância e descrevem um padrão característico da maneira de alternar dos participantes pacientes que contrasta com as características da alternância observada no grupo não-clínico.

Os resultados citados acima refletem dificuldades dos participantes pacientes em uma das dimensões da alternância definida por Mueser et al. (1991), que se refere ao conteúdo descontextualizado ou bizarro da fala. Além disso, os resultados obtidos no presente estudo colocam em destaque outros déficits não incluídos na definição de Mueser et al. (1991), mas que também afetam a alternância da fala, tais como déficits no ritmo da fala, na suficiência do conteúdo da fala e na duração das alternâncias, incluindo as vocalizações. As vocalizações e respostas monossilábicas dos participantes pacientes constituíram o seu padrão de resposta, tendo substituído, portanto, as respostas verbais mais longas, que constituíram, por outro lado, o padrão de resposta do grupo não-clínico. Essas características da alternância apresentadas pelo grupo clínico contribuíram para um desequilíbrio na forma de interagir e de alternar, devendo ser igualmente consideradas ao se estudar o comportamento de alternância. Os resultados obtidos na presente pesquisa contribuíram, portanto, para o estudo da alternância, servindo para ampliar a descrição dos aspectos da alternância a serem focalizados ao se fazer uma avaliação através da observação sistemática do comportamento.

As outras dimensões da alternância que podem ser deficitárias, segundo Mueser et al. (1991), envolvem uma rapidez excessiva do comportamento de alternar de modo a interromper a fala do interlocutor ou, ao contrário, uma longa demora ou latência longa da alternância, criando silêncios entre as falas do sujeito e do interlocutor. Essas duas deficiências no comportamento de alternância também foram observadas na presente pesquisa ao se analisar as reações dos partici- 
pantes aos dois tipos de situações experimentais: de receber e de fazer crítica. Nas situações de receber crítica, observaram-se mais alternâncias atrasadas, com longas latências e com maior freqüência e duração de pausas durante a fala, do que nas situações de fazer crítica, para ambos os grupos, sugerindo hesitação. $O$ grupo não-clínico apresentou, além disso, nas situações de receber críticas, uma menor freqüência de alternâncias com interrupções da fala do interlocutor, um menor número de palavras e uma duração menor das alternâncias do que nas situações de fazer crítica. Esses resultados sugerem a presença de uma maior dificuldade dos participantes nas situações de receber críticas. De fato, a presença de pausas ao falar tem sido utilizada como medida indicadora de ansiedade nas interações sociais (Nelson, Hayes, Felton \& Jarrett, 1985).

Os resultados obtidos na presente pesquisa concordam com dados de Bandeira et al. (2002), nos quais observou-se uma dificuldade maior dos participantes nas situações de receber crítica, em termos dos componentes verbais e não-verbais da assertividade, assim como dados de Bandeira e Ireno (2002), indicando menor impressão global de assertividade nas situações de receber crítica. Esses resultados são também semelhantes aos dados obtidos por Tremblay (1992), nos quais houve maior dificuldade de pacientes psiquiátricos de se comportar de forma socialmente competente nas situações de receber crítica do que nas de fazer crítica. Segundo Argyle (1984), os pacientes psiquiátricos, em particular os esquizofrênicos, mostram-se sensíveis a situações em que são criticados, supervisionados ou questionados, 0 que corrobora os dados obtidos na presente pesquisa.

No que se refere às reações dos participantes ao gênero do interlocutor, os resultados mostraram alternâncias mais longas e no momento adequado, sem interrupções da fala do outro, frente ao interlocutor masculino, para ambos os grupos. Observou-se, ainda, para o grupo não-clínico, uma menor freqüência de alternâncias curtas (com menos de cinco palavras) e monossilábicas para o interlocutor do sexo masculino. Esses resultados concordam com dados obtidos em nosso meio, nos quais se constatou uma maior freqüência de componentes assertivos verbais e não-verbais frente ao interlocutor masculino, tais como expressar mais necessidades ou insatisfação, solicitar mais mudança de comportamento (Bandeira et al., 2002), assim como uma impressão global de maior assertividade frente ao interlocutor masculino (Bandeira \& Ireno, 2002). Esses resultados são coerentes, pois as alternâncias com falas mais longas ocorrem quando há maior grau de assertividade e competência social (Trower, 1980).

O déficit de alternância observado na presente pesquisa para os participantes pacientes confirma dados relatados por outros pesquisadores. Mueser et al. (1991), assim como Argyle e Kendon (1967), observaram que os esquizofrênicos, em particular, possuem déficits na habilidade de alternância, o que pode ter um efeito crítico nas interações sociais, uma vez que limita sua capacidade de comunicação. Mueser et al. (1991) observaram déficit na habilidade de alternância de pacientes psiquiátricos quando comparados com um grupo não-clínico, que se manteve consistente e estável durante todo o período de um ano de observação. Tremblay (1992) observou igualmente um déficit no ritmo da alternância, pois obteve um maior número de pausas ao falar em um grupo de pacientes psiquiátricos, comparativamente a um grupo não-clínico, assim como menor duração da fala e menor grau de competência social.

Sendo a alternância um dos componentes das habilidades sociais, as dificuldades observadas no presente estudo no comportamento de alternar dos participantes pacientes são, portanto, indicadoras de um déficit de competência social. Os resultados obtidos confirmaram essa relação, pois se constatou que quanto maior era o déficit de alternância, menor era o grau de competência social. A impressão global de falta de competência social apresentada pelos participantes pacientes estava relacionada, na análise discriminante, a 
todos os aspectos da alternância estudados na presente pesquisa, e, de forma mais acentuada, com três aspectos: a insuficiência e a descontextualização do conteúdo da fala e sua curta duração. Essa relação entre incompetência social e dificuldades da alternância confirma dados de outros pesquisadores. Segundo Fischetti et al. (1977), as pessoas que apresentam déficits em habilidades sociais geralmente demonstram dificuldades na capacidade de alternância com o interlocutor e os pacientes psiquiátricos têm demonstrado dificuldades nessas habilidades. No estudo de Tremblay (1992), tanto a competência social quanto o comportamento de alternância estavam deficitários no grupo clínico comparativamente a um grupo não-clínico de mesmo nível socioeconômico e do mesmo meio geográfico na comunidade.

Os déficits de alternância, em particular os que se referem ao momento da alternância, podem estar relacionados à presença de dificuldades no processo de percepção social e no processamento de informações dos estímulos que ocorrem em uma interação social (Trower, 1980; Mueser et al., 1991). Por outro lado, os déficits referentes a alternâncias excessivamente curtas ou apenas com vocalizações podem estar relacionados a uma dificuldade de assertividade dos pacientes nas interações sociais (Trower, 1980).

Os participantes pacientes apresentaram igualmente uma menor variabilidade nos parâmetros da alternância estudados na presente pesquisa frente a mudanças no tipo de demanda da situação e no gênero do interlocutor. Esses resultados estão de acordo com dados de outros pesquisadores, segundo os quais os pacientes psiquiátricos apresentam menor variabilidade ou flexibilidade do comportamento frente a mudanças situacionais (Bellack, Morrison, Mueser, Wade \& Sayers, 1990; Moos, 1968). Segundo Trower (1980), essa maior constância do comportamento é decorrente de uma maior dificuldade de discriminar e monitorar variações que ocorrem nos estímulos ambientais, provenientes de mudanças no comportamento dos interlocutores e nas demandas das situações, assim como dificuldade em se automonitorar.

Os resultados desta pesquisa apontam para características do comportamento de alternância dos participantes pacientes que diferem do grupo não- clínico e que se relacionam com a impressão global de baixa competência social. Esses resultados indicam, assim, aspectos específicos a serem focalizados em treinamentos de habilidades sociais nos programas de reabilitação psicossocial que visam melhorar a comunicação dos pacientes psiquiátricos em suas interações sociais.

\section{REFERÊNCIAS}

Argyle, M. (1984). Social skills and the analysis of situations and conversations. In C.R. Hollin \& P. Trower (Org.). Handbook of social skills training: clinical applications and new directions (p.185-216). New York: Pergamon Press.

Argyle, M. (1994) Psicologia del comportamiento interpessoal. Madri: Alianza Universidad.

Argyle, M., \& Kendon, A. (1967). The experimental analysis of social performance. Advances in Experimental Social Psychology, 3, 55-98.

Bandeira, M. (2002). Escala de avaliação da competência social de pacientes psiquiátricos, através de desempenho de papéis. Avaliação Psicológica, 1 (2), 159-171.

Bandeira, M., Cardoso, C.S., Fernandes, M.L., Resende, R.A., \& Santos, S.C.A.S. (1998). Competência social de psicóticos: validação social de habilidades específicas. Jornal Brasileiro de Psiquiatria, 47 (5), 217-226.

Bandeira, M., Gelinas, D., \& Lesage, A. (1998). Desinstitucionalização: o programa de acompanhamento intensivo na comunidade. Jornal Brasileiro de Psiquiatria, 47 (12), 627-640.

Bandeira, M., \& Tremblay, L. (1998). Competência social de psicóticos: um estudo de validação social. Jornal Brasileiro de Psiquiatria, 47 (4), 185-192. 
Bandeira, M. \& Ireno, E.M. (2002). Reinserção social de psicóticos: avaliação global do grau de assertividade, em situações de fazer e receber crítica. Psicologia: Reflexão e Crítica, 15 (3), 665-675.

Bandeira, M., Machado, E.L., \& Pereira, E.A. (2002). Reinserção social de psicóticos: componentes verbais e não-verbais do comportamento assertivo em situações de fazer e receber críticas. Psicologia: Reflexão e Crítica, 15 (1), 89-104.

Bellack, A.S., Morrison, R.L., Mueser, K.T., \& Wade, J.H. (1989). Social competence in schizoaffective disorder, bipolar disorder, and negative and non-negative schizophrenia. Schizophrenia Research, 2, 391-401.

Bellack, A.S., Morrison, R.L., Mueser, K.T., Wade, J.H., \& Sayers, S. L. (1990). Role play for assessing the social competence of psychiatric patients. Journal of Consulting and Clinical Psychology, 2 (3), 248-255.

Donahoe, C.P., \& Driesienga, S.A. (1988). A Review of social skills training with chronic mental patients. In M. Hersen, R.M. Eisler \& P.M. Miller (Org.). Progress in Behavioral Modification (v. 23, p.131-164). Newbury Park, CA: Sage.

Fischetti, M., Curran, J.P., \& Wessberg, H.W. (1977). Sense of timing: a skills deficit in heterosexual- socially anxious males. Behavior Modification, 1 (2), 179-195.

Hogarty, G.E., Anderson, C.M., Reiss, D.J., Kornblith, S.J., Greenwald, D.P., Ulrich, R.F., \& Carter, M. (1991). Family psychoeducation, social skills training, and mainterance chemotherapy in the aftercare treatment of schizophrenia. Archives of General Psychiatry, 48, 340-347.

Kazdin, A.E. (1977). Assessing the clinical or applied importance of behavior change through social validation. Behavior Modification, 1, 427-451.

Kendon, A. (1967). Some functions of gaze direction in social interaction. Acta Psychology, 26, 22-63.
Liberman, R.P. (1984). Social skills training with relapsing schizophrenics: an experimental analysis. Behavior Modification, 8, 155-173.

Liberman, R.P. (1986). Psychiatric rehabilitation of schizophrenia: editor's introduction. Schizophrenia Bulletin, 12, 540-541.

Liberman, R.P., Wallace, C.J., Blackwell, C.A., Eckman, T.A., Vaccaro, J.V., \& Kuehnel, T.G. (1993). Innovations in skills training for the seriously mentally ill: the ucla social and independent living skills modules. Innovations and Research, 2, 43-60.

Merluzzi, T.V., \& Biever, J. (1987). Role-playing procedures for the behavioral assessment of social skill: a validity study. Behavioral Assessment, 9, 361-377.

Moos, R.H. (1968). Situacional analysis of a therapeutic community milieu. Journal of Abnormal Psychology, 73 (1), 49-61.

Mueser, K.T., Bellack, A.S., Douglas, M.S., \& Morrison, R.L. (1991). Prevalence and a stability of social skills deficits in schizophrenia. Schizophrenia Research, 5, 167-176.

Nelson, R.O., Hayes, S.C., Felton, J.L., \& Jarret, R.B. (1985). A comparison of data produced by different behavioral assessment techniques with implications for models of social-skills inadequacy. Behavior Research and Therapy, 23 (1), 1-11.

Stein, L.I. \& Test, M.A. (1980). Alternative to mental hospital treatment: conceptual model, treatment program and clinical evaluation. Acta Gen Psychiatry, 37 ( 4), 392-397.

Torgrud, L.J., \& Holborn, S.W. (1992). Developing externally valid role-play for assessment of social skills: a behavior analytic perspective. Behavioral Assessement, 14, 245-277.

Tremblay, L. (1992). Évaluation de la competence sociale de personnes souffrant de troubles mentaux graves: études de validation sociale. Relatório $\mathrm{n}$. RS-1725-089. Conseil Québecois de la Recherche Sociale, Montréal. 
Tremblay, L., \& Bandeira, M. (1989). L'Evaluation ecologique des habiletes sociales (EEHS). Document Inedit de L Institut Pinel de Montreal.

Trower, P. (1980). Situational analysis of the components and processes of behavior of socially skilled and unskilled patients. Journal of Consul and Clinical Psychology, 48, 327-339.
Wallace, C.J., Liberman, R.P., Mackain, S.J., Blackwell, G., \& Eckman, T.A. (1992). Effectiveness and replicability of modules for teaching social and instrumental skills to the severely mentally ill. American Journal of Psychiatry, 149 (5), 654-658.

Recebido para publicação em 12 de fevereiro de 2003 e aceito em 28 de outubro de 2004. 
